\title{
Proteomic comparison between abdominal and thoracic aortic aneurysms
}

\author{
KEN-ICHI MATSUMOTO ${ }^{1}$, KAZUMI SATOH ${ }^{1}$, TOMOKO MANIWA $^{1}$, \\ TETSUYA TANAKA $^{2}$, HIDEKI OKUNISHI ${ }^{2}$ and TEIJI ODA ${ }^{3}$
}

\begin{abstract}
${ }^{1}$ Department of Biosignaling and Radioisotope Experiment, Interdisciplinary Center for Science Research, Organization for Research, Shimane University, ${ }^{2}$ Department of Pharmacology and ${ }^{3}$ Division of Cardiovascular and Thoracic Surgery, Department of Surgery, Shimane University School of Medicine, Izumo 693-8501, Japan
\end{abstract}

Received September 28, 2013; Accepted January 14, 2014

DOI: $10.3892 /$ ijmm.2014.1627

\begin{abstract}
The pathogenesis of abdominal aortic aneurysms (AAAs) and that of thoracic aortic aneurysms (TAAs) is distinct. In this study, to reveal the differences in their biochemical properties, we performed quantitative proteomic analysis of AAAs and TAAs compared with adjacent normal aorta (NA) tissues. The proteomic analysis revealed 176 non-redundant differentially expressed proteins in the AAAs and 189 proteins in the TAAs which were common in at least 5 samples within 7 samples of each. Among the identified proteins, 55 and 68 proteins were unique to the AAAs and TAAs, respectively, whereas 121 proteins were identified in both the AAAs and TAAs. PANTHER overrepresentation analysis of the unique proteins in the AAAs and TAAs revealed a significant downregulation of the blood coagulation pathway in the AAAs and that of the integrin signaling pathway in the TAAs. On the other hand, Genesis analysis revealed distinct expression patterns of 58 proteins among the 121 proteins. PANTHER overrepresentation analysis of these 58 proteins revealed that the expression of these proteins in the blood coagulation and the plasminogen activating cascade was decreased in the AAAs, whereas it was increased in the TAAs compared with the NA tissues. On the other hand, the protein expression in the integrin signaling pathway was increased in the AAAs, whereas it was decreased in the TAAs compared with the NA tissues. Thus, the data presented in this study indicate that the proteins that show differential expression patterns in AAAs and TAAs may be involved in the distinct pathogenesis of AAAs and TAAs.
\end{abstract}

Correspondence to: Dr Ken-Ichi Matsumoto, Department of Biosignaling and Radioisotope Experiment, Interdisciplinary Center for Science Research, Organization for Research, Shimane University, 89-1 Enya-cho, Izumo 693-8501, Japan

E-mail: matumoto@med.shimane-u.ac.jp

Key words: abdominal aortic aneurysm, thoracic aortic aneurysm, isobaric tag for relative and absolute quantitation, proteomics

\section{Introduction}

An aortic aneurysm (AA) is an enlargement which occurs in the aorta, leading to progressive dilatation and ultimate rupture. According to their anatomical locations, AAs are generally classified as abdominal AAs (AAAs) and thoracic AAs (TAAs), which appear to have distinct pathologies and mechanisms (1). AAAs are much more common (an incidence of at least 3-fold higher) than TAAs (2). The differences in the physical structure and mechanical stress of abdominal and thoracic aortas may also contribute to the disparities in the pathogenesis of AAAs and TAAs (3). Atheroscrelosis is well known to be associated with AAAs, whereas it rarely occurs in patients with TAAs. The increased expression of growth factors, inflammatory cytokines and matrix metalloproteinses (MMPs) induces the migration of macrophages and $\mathrm{T}$ cells and the formation of atherosclerotic plaques. MMPs contribute to progressive structural remodeling, particularly elastin and collagen turnover in AAAs. The apoptosis of vascular smooth muscle cells (VSMCs) has also been demonstrated in the media layer of AAA walls (4). These changes are associated with the progressive weakening of the aortic walls in AAAs. Furthermore, several studies have suggested that Th2-type immune responses play a major role in the formation of AAAs (1). The levels of Th-2 cytokines, such as interleukin (IL)-4, IL-5 and IL-10 are also significantly increased in AAAs.

On the other hand, genetic contribution to TAAs (approximately $20 \%$ of TAAs) is greater than that to AAAs. Connective tissue disorders, such as Marfan syndrome, Loeys-Dietz syndrome and Ehlers-Danlos syndrome type IV, influence the pathogenesis of TAAs. Medial degeneration associated with the loss of VSMCs and the destruction of medial elastic fibers is observed much more frequently in patients with TAAs than in patients with AAAs. As regards inflammation and cellular immune responses in TAAs, Th1-type immune responses generally predominate in TAAs (5). The increased expression of the Th1 cytokine, interferon- $\gamma$, in TAAs has also been demonstrated.

A number of studies have revealed the proteomic or transcriptional profile associated with the pathological remodeling process in AAAs or TAAs $(6,7)$. However, to the best of our 
knowledge, a comparison of the proteome spectrum occurring in AAAs and that occurring in TAAs has not been carried out to date.

In a recent study of ours (8), we successfully applied quantitative proteomic analyses using tandem mass spectrometry (MS) with an isobaric tag for relative and absolute quantitation (iTRAQ) labeling strategy to reveal differentially expressed proteins in diseased tissues and adjacent normal tissues. The proteomic analyses revealed differentially expressed proteins in calcified AAAs (CAAs) and calcified TAAs (CTAs) in contrast to adjacent normal tissues (8). Consequently, the proteins involved in aneurysm formation and vascular calcification were identified (e.g., type I and type III collagen, matrix Gla protein and $\alpha$-2-HS-glycoprotein in CAAs; fibrinogen chains and $\alpha$-2-HS-glycoprotein in CTAs with an increased expression; mimecan in CAAs; and fibulin-5 in CTAs with a decreased expression compared with adjacent normal tissues). Furthermore, proteomic analysis of differentially expressed proteins in calcific aortic valves (CAVs) compared with those in adjacent normal valvular tissues revealed that $\alpha$-2-HSglycoprotein had the greatest increase in expression and that tenascin-X had the greatest decrease in expression in the CAVs (9).

In this study, in order to elucidate the distinct spectrum of molecular alteration leading to the differences in the pathology between AAAs and TAAs, we attempted to reveal differentially expressed proteins in AAAs and TAAs compared with adjacent normal aorta (NA) tissues using the iTRAQ technology (10), followed by nano-liquid chromatography (nano-LC)-matrix-assisted laser desorption ionization (MALDI)/time-of-flight (TOF/TOF)-tandem MS/MS $(8,11)$. We then compared the differentially expressed proteins in AAAs with those in TAAs.

\section{Patients and methods}

Patients and samples. Aortic aneurysm tissues were collected after obtaining approval from the Ethics Committee of Shimane University School of Medicine, Izumo, Japan. All study participants provided informed consent. Aortic aneurysm tissues for quantitative differential expression analysis were obtained at surgery from 7 patients with AAAs [5 males and 2 females; age, 60-87 years; average age (means \pm SD), $76.6 \pm 10.5$ years; size of aneurysms, 45-60 (52.9 \pm 5.9$) \mathrm{mm}]$ and from 7 patients with TAAs [4 males and 3 females; age, 63-87 years; average age, $78.6 \pm 8.3$ years; size of aneurysms, 53-72 $(61.3 \pm 6.3) \mathrm{mm}$ ] who underwent aortic aneurysm resection in Shimane University Hospital. Control samples were obtained from relatively NA tissues adjacent to the AAA or TAA tissues. In addition, another 10 AAA and 10 TAA and corresponding adjacent NA tissues for confirmation by western blot analysis were obtained from 10 patients with AAAs [ 8 males and 2 females; age, 62-89 years; average age, $78.5 \pm 7.4$ years; size of aneurysms, 40-74 $(55.2 \pm 10.1) \mathrm{mm}]$ and 10 patients with TAAs [6 males and 4 females; age, 55-80 years; average age, 70.6 \pm 7.9 years; size of aneurysms, 45-70 (55.9 \pm 7.6$) \mathrm{mm}$. The sample number, gender, age and size of aneurysms are shown in Table I. Tissue samples were snap-frozen in liquid nitrogen and stored at $-80^{\circ} \mathrm{C}$ until protein extraction.
Table I. Samples used for MS analysis and western blot analysis.

Size of aneurysm

Gender Age (mm)

Samples used for iTRAQ labeling followed by MS analysis AAA patient no.

$\begin{array}{lccc}\text { AAA1 } & \text { M } & 60 & 50 \\ \text { AAA2 } & \text { F } & 80 & 58 \\ \text { AAA3 } & \text { M } & 87 & 46 \\ \text { AAA4 } & \text { M } & 81 & 60 \\ \text { AAA5 } & \text { M } & 64 & 45 \\ \text { AAA6 } & \text { M } & 78 & 55 \\ \text { AAA7 } & \text { F } & 86 & 56 \\ \text { TAA patient no. } & & & \\ \text { TAA1 } & \text { M } & 78 & 62 \\ \text { TAA2 } & \text { M } & 87 & 72 \\ \text { TAA3 } & \text { F } & 78 & 60 \\ \text { TAA4 } & \text { F } & 75 & 63 \\ \text { TAA5 } & \text { M } & 63 & 53 \\ \text { TAA6 } & \text { F } & 87 & 55 \\ \text { TAA7 } & \mathrm{M} & 82 & 64\end{array}$

Samples from other patients used for the determination of TNC expression by western blot analysis

AAA patient no.

$\begin{array}{llll}\text { AAA8 } & \text { M } & 77 & 58 \\ \text { AAA9 } & \text { M } & 62 & 63 \\ \text { AAA10 } & \text { M } & 77 & 62 \\ \text { AAA11 } & \text { M } & 81 & 47 \\ \text { AAA12 } & \text { M } & 81 & 40 \\ \text { AAA13 } & \text { M } & 83 & 51 \\ \text { AAA14 } & \text { F } & 79 & 57 \\ \text { AAA15 } & \text { F } & 84 & 74 \\ \text { AAA16 } & \text { M } & 89 & 56 \\ \text { AAA17 } & \text { M } & 72 & 44\end{array}$

TAA patient no.

$\begin{array}{lccc}\text { TAA8 } & \text { F } & 80 & 62 \\ \text { TAA9 } & \text { M } & 78 & 70 \\ \text { TAA10 } & \text { M } & 78 & 58 \\ \text { TAA11 } & \text { M } & 75 & 54 \\ \text { TAA12 } & \text { F } & 66 & 54 \\ \text { TAA13 } & \text { F } & 64 & 47 \\ \text { TAA14 } & \text { M } & 65 & 52 \\ \text { TAA15 } & \text { M } & 74 & 54 \\ \text { TAA16 } & \text { F } & 71 & 45 \\ \text { TAA17 } & \text { M } & 55 & 63\end{array}$

iTRAQ, isobaric tag for relative and absolute quantitation; MS, mass spectrometry; AAA, abdominal aortic aneurysms; TAA, thoracic aortic aneurysms; TNC, tenascin-C.

Sample preparation. Sample preparation was carried out according to the manual supplied by AB Sciex (Foster City, CA, USA) and according to our previous studies $(8,9)$. Briefly, 
$\sim 30 \mathrm{mg}$ of each of the AAA or TAA tissues and their adjacent NA tissues as controls were subjected to protein extraction. Following the addition of urea lysis buffer containing $7 \mathrm{M}$ urea, $0.1 \%$ Nonidet P-40 (NP-40) and $500 \mathrm{mM}$ triethylammonium bicarbonate (TEAB) (Sigma, Tokyo, Japan), the sample was sonicated, incubated at $4^{\circ} \mathrm{C}$ for $1 \mathrm{~h}$, and centrifuged, and then the supernatant was collected. The supernatant was desalted and its buffer was exchanged with $50 \mathrm{mM}$ TEAB using spin concentrators (Corning, Tokyo, Japan). The protein concentration was determined using a bicinchoninic acid (BCA) assay kit (Thermo Fisher Scientific, Waltham, MA, USA).

iTRAQ labeling. Labeling with iTRAQ was carried out using iTRAQ $^{\mathrm{TM}}$ Reagent from AB Sciex as described in the manual supplied. First, $125 \mu \mathrm{g}$ of proteins in each of the lysates from the AAA or TAA samples and adjacent NA samples were denatured by sodium dodecyl sulfate (SDS) and reduced by [tris-(2-carboxyethyl)phosphine (TCEP)]. Cysteine alkylation was then carried out by methyl methanethiosulfonate (MMTS). Each sample from the AAA or TAA and adjacent NA tissues was digested by trypsin. Each digest was labeled with a different iTRAQ tag using an iTRAQ Reagent Multiplex kit (AB Sciex). The labeled AAA or TAA and control samples were then combined. The combined samples were fractionated into 6 fractions with a strong cation exchange (SCX) chromatograph (AB Sciex) according to the manufacturer's instructions. Each of the fractions was then desalted by a Sep-Pac $\mathrm{C}_{18}$ cartridge (Waters Corp., Milford, MA, USA).

Nano-LC. Fractionation with the DiNa nano-LC system was performed according to the instructions provided by the manufacturer (KYA Technologies, Tokyo, Japan) and our previous study (11). A total of 171 spots that were mixed directly with a matrix [4 $\mathrm{mg} / \mathrm{ml} \alpha$-cyano-4-hydroxycinnamic acid (CHCA); Wako, Osaka, Japan] were placed on an Opti-TOF LC/MALDI 384 target plate (AB Sciex) using a Dina MaP fraction collector (KYA Technologies) per fraction of SCX chromatography.

MS, MS/MS and iTRAQ ratio analysis. MS data were obtained using a Mass Spectrometer 5800 MALDI-TOF/TOF Analyzer (AB Sciex) according to the instructions provided by the manufacturer (AB Sciex) and our previous study (11). A monoisotopic precursor for MS/MS was selected by automatic precursor selection with an interpretation method using the DynamicExit Algorithm (AB Sciex). The MS/MS data were analyzed by ProteinPilot ${ }^{\mathrm{TM}} 3.0$ software using the Paragon protein database search algorithm (AB Sciex), as previously described (12). Each MS/MS spectrum was searched against the database (version 20081216, 20,489 entries) constructed by AB Sciex. The statistical method of iTRAQ analysis was according to ProteinPilot software.

Bioinformatic analysis. The PANTHER system (http://www. pantherdb.org/) was used for classification and pathway analyses of proteins (13). Pathway classification analysis was carried out using a statistical overrepresentation test on PANTHER with Bonferroni correction for multiple testing. The UniProt database (http://www.uniprot.org/) was used for annotations of identified proteins. Clustering analysis of differentially expressed proteins was performed using Genesis software provided by the Genesis team at the Institute for Genomics and Bioinformatics, Graz University of Technology (Graz, Austria) (http://genome.tugraz.at/) (14).

Western blot analysis. Cell lysates were extracted with urea lysis buffer as described above. Western blot analysis was performed as described in our previous study (8). A total of $20 \mu \mathrm{g}$ of proteins in these lysates was electrophoresed through sodium dodecyl sulfate (SDS)-polyacrylamide gel electrophoresis (PAGE), and then the proteins were transferred onto Hybond ECL nitrocellulose membranes (GE Healthcare Japan, Hino, Japan). The membranes were reacted with rabbit polyclonal anti- $\alpha-2-H S$-glycoprotein antibody (Calbiochem, Darmstadt, Germany), rabbit polyclonal anti-ceruloplasmin antibody (Epitomics, Burlingame, CA, USA) or mouse monoclonal anti-tenascin-C (TNC) antibody [Immuno Biological Laboratories (IBL) Takasaki, Japan]. The proteins on the membranes were then reacted with anti-rabbit IRDye 680-conjugated immunoglobulin (Ig)G or anti-mouse IRDye 800-conjugated $\mathrm{IgG}$, followed by visualization using the infrared imaging system, Odyssey (all from LI-COR Biosciences, Lincoln, NE, USA). For densitometric analyses of each protein level, the intensity of each band that reacted with the corresponding antibody was measured. Data from triplicate experiments were analyzed for statistical significance by the paired t-test, with $\mathrm{p}<0.05$ considered to indicate a statistically significant difference. Results are expressed as the means \pm standard error (SE).

\section{Results}

Proteomic analyses of differentially expressed proteins in AAAs or TAAs compared with those in adjacent NA tissues. A total of 7 AAAs and 7 TAAs, as well as adjacent NA tissues were collected from 7 AAA and 7 TAA patients. The identification and quantification of differentially expressed proteins in AAAs or TAAs compared with those in adjacent NA tissues were carried out with iTRAQ labeling coupled with nano-LC-MALDI-TOF/TOF-MS/MS followed by ProteinPilot analysis. Apart from albumin and Ig family members, a total of 656 differentially expressed proteins (AAAs vs. NA tissues) in at least 1 sample within 7 AAAs and 654 differentially expressed proteins (TAAs vs. NA tissues) in at least 1 sample within 7 TAAs were identified. Among these, 483 proteins were detected in both aneurysmal tissues (Fig. 1A). A total of 176 differentially expressed proteins (AAAs vs. NA tissues) which were common in at least 5 samples within 7 AAAs and 189 differentially expressed proteins (TAAs vs. NA tissues) which were common in at least 5 samples within 7 TAAs were identified with an unused ProtScore of $\geq 2$ (99\% confidence) based on ProteinPilot statistical analysis. Among these, 121 proteins were detected which were common in both aneurysmal tissues (Fig. 1B). Of note, after having classified the patients based on the diameter of the aneurysms (small group, $\leq 55 \mathrm{~mm}$; large group, $>55 \mathrm{~mm}$ ) (small diameter group, \#AAA1, \#AAA3, \#AAA5 and \#AAA6; large diameter group, \#AAA2, \#AAA4 and \#AAA7 in the case of AAA; small diameter group, \#TAA5 and \#TAA6; large diameter group, \#TAA1, \#TAA2, \#TAA3, \#TAA4 and \#TAA7 in the case of TAA), we examined which proteins in the 176 AAA and 


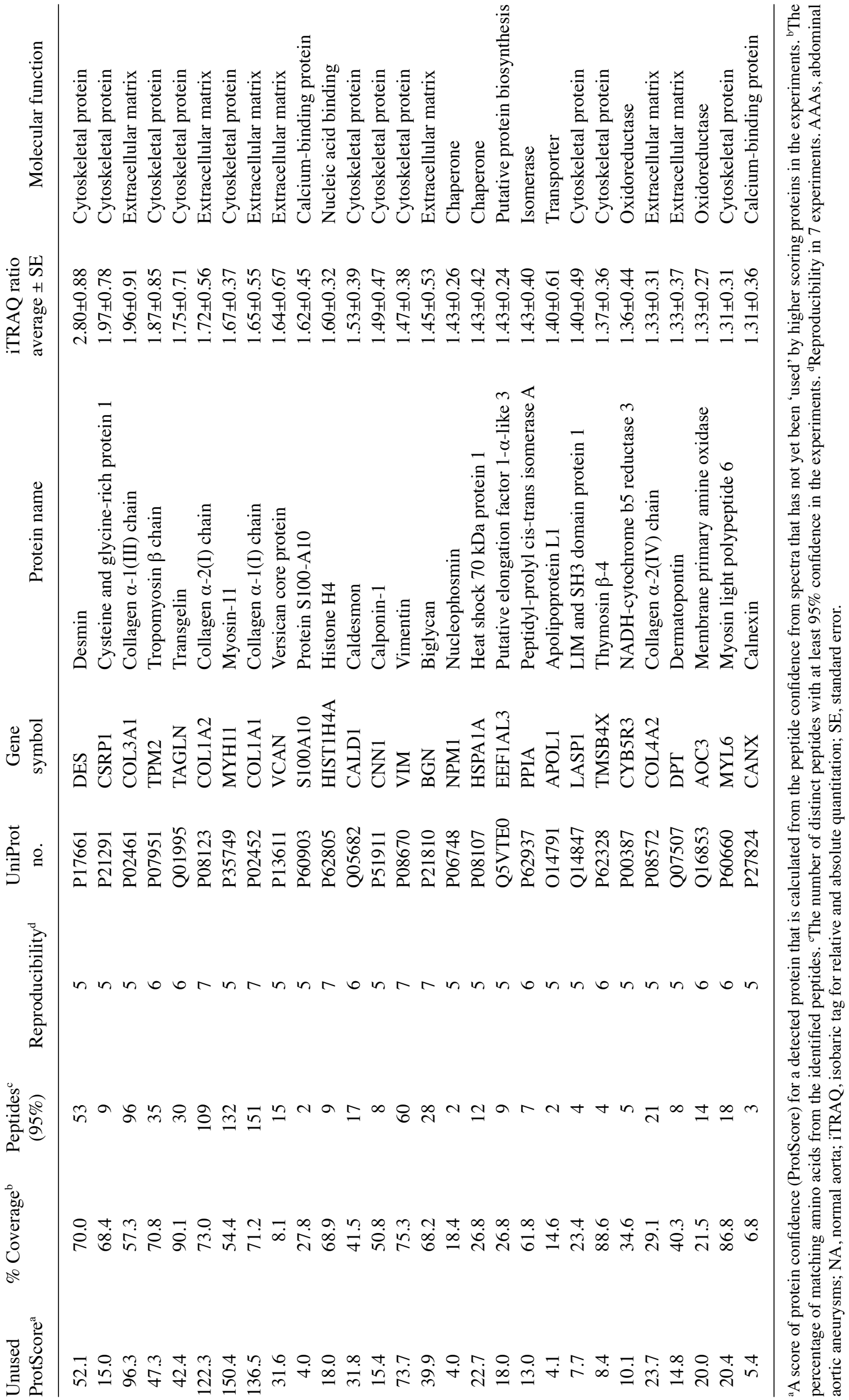


A

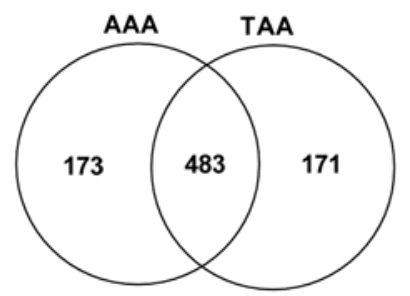

B

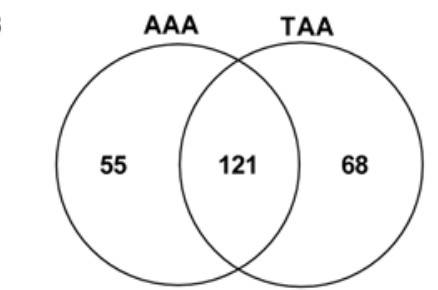

\begin{tabular}{|c|c|c|}
\hline \multirow{2}{*}{ iTRAQ ratio } & \multicolumn{2}{|c|}{ Number of proteins } \\
\cline { 2 - 3 } & AAA & TAA \\
\hline$\geqq 1.3$ & 28 & 18 \\
\hline intermediate & 136 & 28 \\
\hline$<0.77$ & 12 & 143 \\
\hline
\end{tabular}

Figure 1. Venn diagrams showing numbers of differentially expressed proteins in abdominal aortic aneurysms (AAAs), thoracic aortic aneurysms (TAAs), and both aneurysms compared with adjacent normal aorta (NA) tissues. (A) Numbers of proteins that were identified in at least 1 sample among 7 samples with isobaric tag for relative and absolute quantitation (iTRAQ) ratios in AAAs and TAAs. (B) Numbers of proteins that were identified in at least 5 samples among 7 samples with iTRAQ ratios in AAAs and TAAs. (C) Numbers of proteins with significantly increased $(\geq 1.3$-fold), intermediate $(\geq 0.77$ and $<1.3$ ) and decreased expression ratios $(<0.77$-fold) that were identified in at least 5 samples among 7 samples in AAAs and TAAs.

189 TAA proteins showed distinct expression patterns in the large diameter group and small diameter group with one-way ANOVA using Genesis software. Consequently, with $\mathrm{p}<0.01$ (small diameter group vs. large diameter group), histone $\mathrm{H} 4$ and nucleophosmin were identified in the AAA proteins, whereas $\alpha$-actinin-4, $\alpha$-1-acid glycoprotein 1 , basement membranespecific heparan sulfate proteoglycan core protein, caldesmon, calreticulin, filamin-A, lamin-A/C, lipoma-preferred partner, $\mathrm{NADH}$-cytochrome b5 reductase 3 , membrane primary amine oxidase, myosin-9, myosin-10, myosin-11, myosin light polypeptide 6 , polymerase I and transcript release factor, transgelin, tropomyosin $\beta$ chain, versican core protein and vimentin were identified in the TAA proteins.

Furthermore, since most biochemical methods tend to have technical variation, we considered an additional cut-off value (1.3-fold change in expression; upregulated proteins, $\geq 1$. 3 -fold change in expression; downregulated proteins, $<0.77$-fold change in expression, AA tissues vs. NA tissues) for iTRAQ ratios for the selection of proteins with differential expression levels, as previously described $(9,15,16)$. As a result, 28 and 18 proteins with a significantly increased expression ( $\geq 1.3$-fold, AA tissues vs. NA tissues) and 12 and 143 proteins with a significantly decreased expression $(<0.77$-fold, AA tissues vs. NA tissues) were identified in the AAAs and TAAs, respectively (Fig. 1C). In Tables II-V, proteins with increased ( $\geq 1.3$-fold, AA tissues vs. NA tissues) and decreased

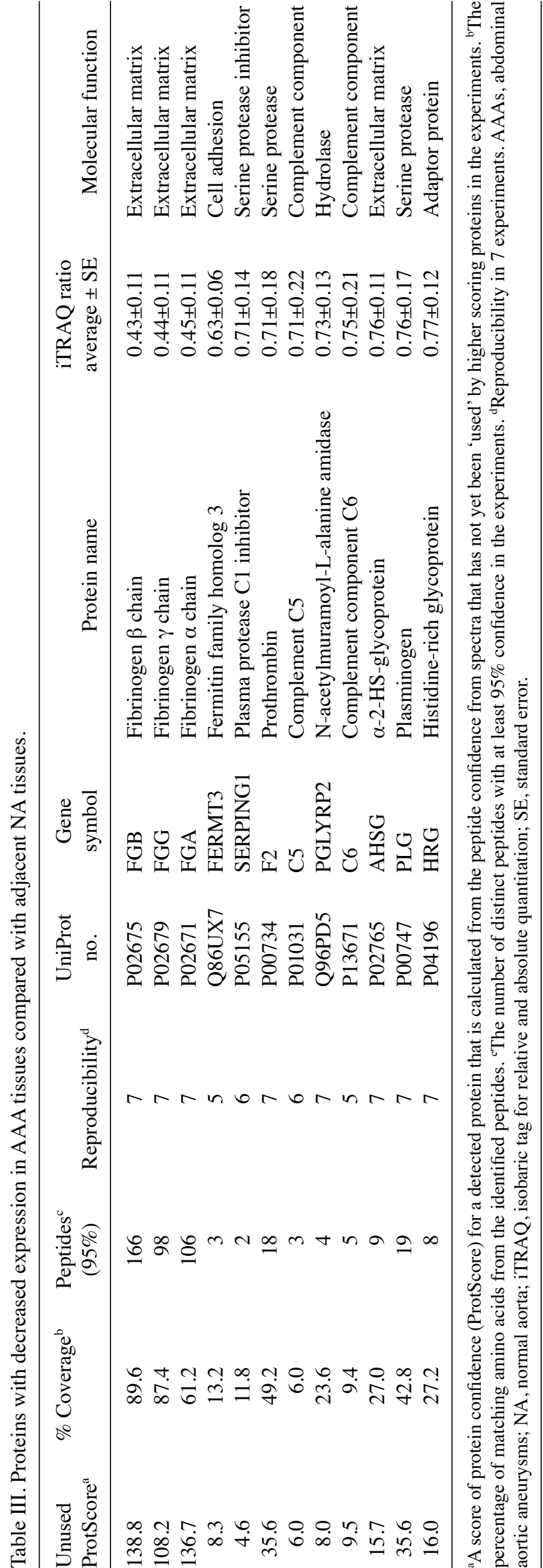




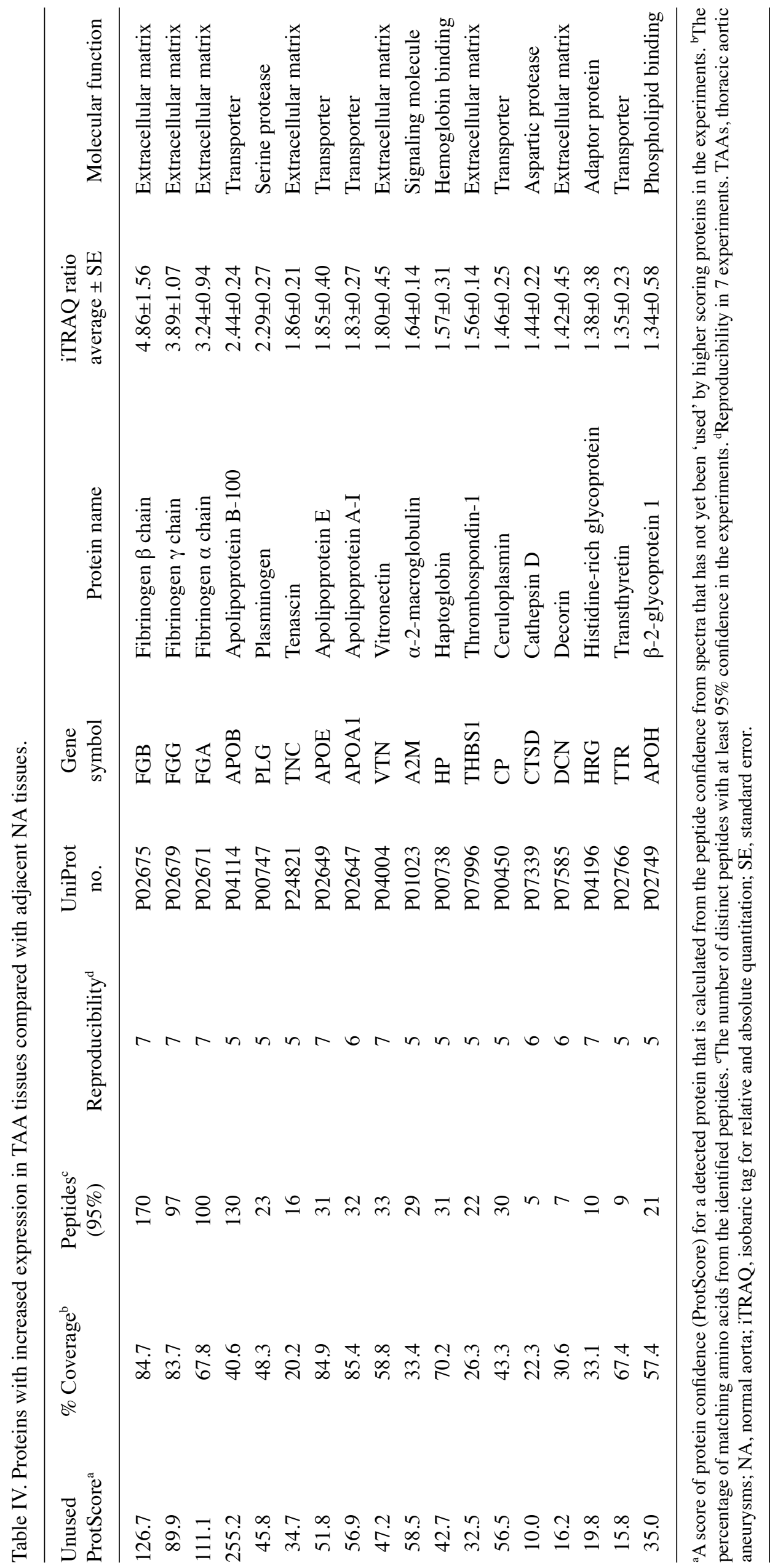




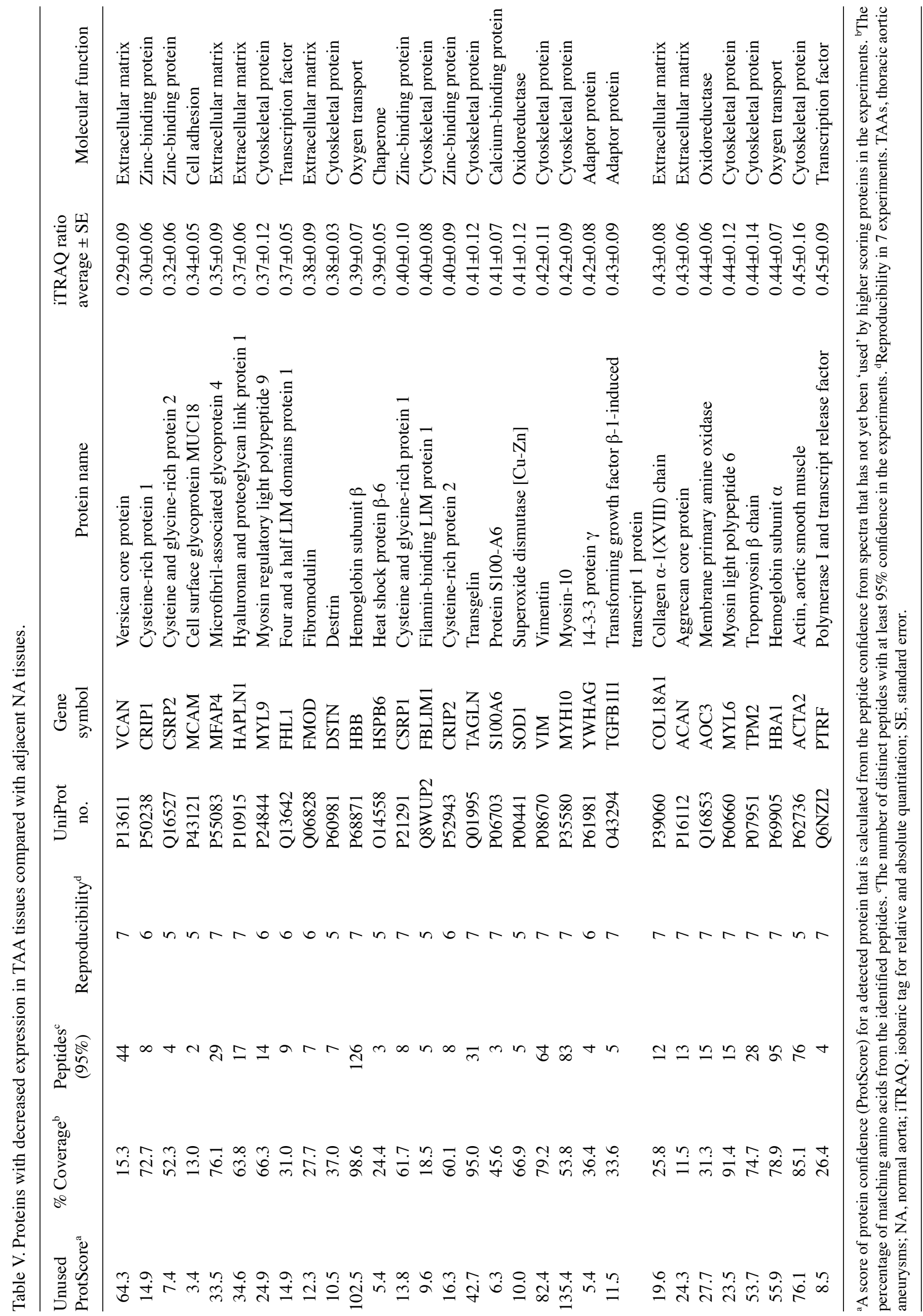


A a

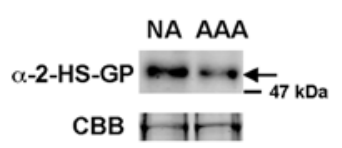

b

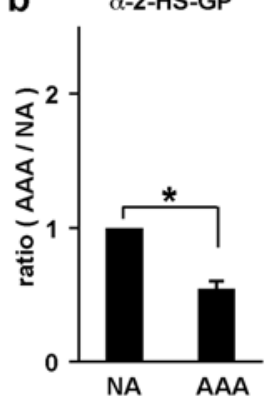

B a

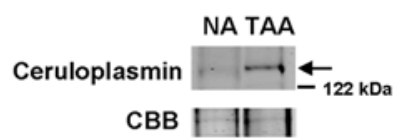

b

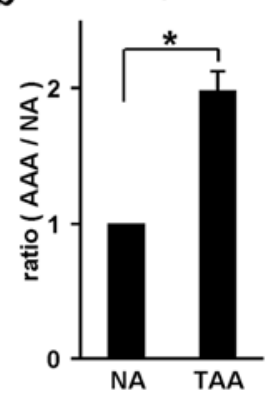

Figure 2. Confirmation of isobaric tag for relative and absolute quantitation (iTRAQ) ratios by western blot analysis. (A-a) Western blot analysis with anti- $\alpha$ 2-HS-glycoprotein antibody in abdominal aortic aneurysms (AAAs) compared with adjacent normal aorta (NA) tissues in patient \#AAA3. To confirm equal levels of proteins per lane, non-specific proteins stained with Coomassie brilliant blue (CBB) are shown in the lower panel. Representative blots are shown. (B-b) Densitometric analyses in triplicate experiments for $\alpha$-2-HS-glycoprotein. The intensity of each band that reacted with $\alpha-2$-HS-glycoprotein antibody was measured. Ratio of expression of $\alpha-2-H S$-glycoprotein in AAAs compared with that in adjacent NA tissues (1.0) was calculated. * $\mathrm{p}<0.05$. (B-a) Western blot analysis with anti-ceruloplasmin antibody in thoracic aortic aneurysms (TAAs) compared with adjacent NA tissues in patient \#TAA4. To confirm equal levels of proteins per lane, non-specific proteins stained with CBB are shown in the lower panel. Representative blots are shown. (B-b) Densitometric analyses in triplicate experiments for ceruloplasmin. The intensity of each band that reacted with ceruloplasmin antibody was measured. Ratio of expression of ceruloplasmin in TAAs compared with that in adjacent NA tissues (1.0) was calculated. ${ }^{*} \mathrm{p}<0.05$.

expression (<0.77-fold, AA tissues vs. NA tissues) in the AAAs and TTAs compared with the adjacent NA tissues are listed in order of iTRAQ ratios.

In order to confirm the accuracy of the quantitative results for the differentially expressed proteins identified, some proteins with an iTRAQ quantitative ratio were quantified again by western blot analysis (Fig. 2). The iTRAQ ratio of $\alpha-2-H S$ glycoprotein in AAAs compared with that in adjacent NA tissues in patient \#AAA3 was 0.79-fold. On the other hand, the ratio of $\alpha$-2-HS-glycoprotein in the AAAs compared with that in NA tissue (1.0) was determined by band intensity of western blot analysis and it was 0.55 -fold (Fig. 2A). Similarly, the iTRAQ ratio of ceruloplasmin in TAAs compared with that in adjacent NA tissues in patient \#TAA4 was 2.25-fold, whereas the ratio of ceruloplasmin based on western blot analysis was 1.98-fold (Fig. 2B). These results indicate that iTRAQ ratios are almost consistent with the quantitative results by western blot analysis.

Analysis of proteins unique to AAAs and TAAs. To disclose distinct molecular alterations that occurred in AAAs and TAAs, the 55 and 68 proteins unique to AAAs and TAAs among the 176 AAA proteins and 189 TAA proteins found in common in at least 5 samples within each of the 7 samples, respectively, were selected (Fig. 1B). On the other hand, 121 differentially expressed proteins were identified which were common in the AAA and TAA samples (Fig. 1B).

We investigated the possible biochemical pathway for the 55 and 68 proteins unique to AAAs and TAAs by using the PANTHER pathway system (Fig. 3). As a result, we found the possible involvement of the blood coagulation pathway (statistical overrepresentation test in PANTHER, $\mathrm{p}=1.53 \mathrm{E}-06$ ) in AAAs. This pathway included coagulation factor XIII A chain (iTRAQ ratio, 1.26, AAAs vs. NA tissues), kininogen-1 (0.88), vitamin K-dependent protein $S(0.85)$, antithrombin-III (0.83), heparin cofactor $2(0.80)$ and prothrombin (0.71); the majority of these proteins were downregulated in the AAA tissues compared with the adjacent NA tissues. On the other hand, in the TAAs, we observed the downregulation of the integrin signaling pathway (statistical overrepresentation test in PANTHER, $\mathrm{p}=6.17 \mathrm{E}-06$ ), including laminin subunit $\gamma-1$ (iTRAQ ratio, 0.65, TAAs vs. NA tissues), integrin-linked protein kinase (ILK) (0.62), caveolin-1 (0.58), type IV collagen $\alpha 1$ chain (0.56), laminin subunit $\beta 2(0.56)$, laminin subunit $\alpha 5$ (0.54), Ras-related protein R-Ras (0.52), $\alpha$-actinin-4 (0.51), transforming growth factor $\beta$-1-induced transcript 1 protein $(0.43)$ in the TAA tissues compared with the adjacent NA tissues. Furthermore, in order to determine the functional distribution of these unique proteins in the AAAs and TAAs, we sorted the proteins with the PANTHER protein class system (Fig. 4). In the AAAs, the 55 unique proteins were significantly classified into defense/immunity protein (statistical overrepresentation test in PANTHER, $\mathrm{p}=5.55 \mathrm{E}-05)$, including complement component $(\mathrm{p}=4.67 \mathrm{E}-10)$, transfer/carrier protein $(\mathrm{p}=4.83 \mathrm{E}-06)$, including apolipoprotein $(\mathrm{p}=2.26 \mathrm{E}-11)$, enzyme modulator including protease inhibitor $(\mathrm{p}=7.56 \mathrm{E}-07)$ and serine protease inhibitor $(\mathrm{p}=6.10 \mathrm{E}-06)$ and protease, including metalloprotease $(p=0.0052)$ and serine protease ( $\mathrm{p}=0.0053)$; the majority of these proteins were downregulated in the AAA tissues compared with the adjacent NA tissues. On the other hand, in the TAAs, the 68 proteins were significantly classified into cytoskeletal protein $(\mathrm{p}=1.21 \mathrm{E}-08)$, including actin family cytoskeletal protein $(p=1.29 \mathrm{E}-14)$ and non-motor actin binding protein $(\mathrm{p}=4.95 \mathrm{E}-03)$, cell adhesion molecule $(p=6.37 \mathrm{E}-04)$, structural protein $(p=1.63 \mathrm{E}-07)$, extracellular matrix (ECM) protein $(\mathrm{p}=7.56 \mathrm{E}-05)$ and calciumbinding protein $(\mathrm{p}=7.31 \mathrm{E}-04)$; the majority of these proteins were downregulated in the TAAs compared with the adjacent NA tissues.

Analysis of 121 differentially expressed proteins identified which were common in AAAs and TAAs. We performed hierarchical clustering analysis with Genesis software using the expression patterns of the 121 differentially expressed proteins identified which were common in at least each of 5 samples within the 7 AAAs and 7 TAAs compared with the corresponding adjacent NA tissues. Among the 121 proteins, we examined proteins that showed statistically distinct expression patterns between the AAAs and TAAs by one-way 


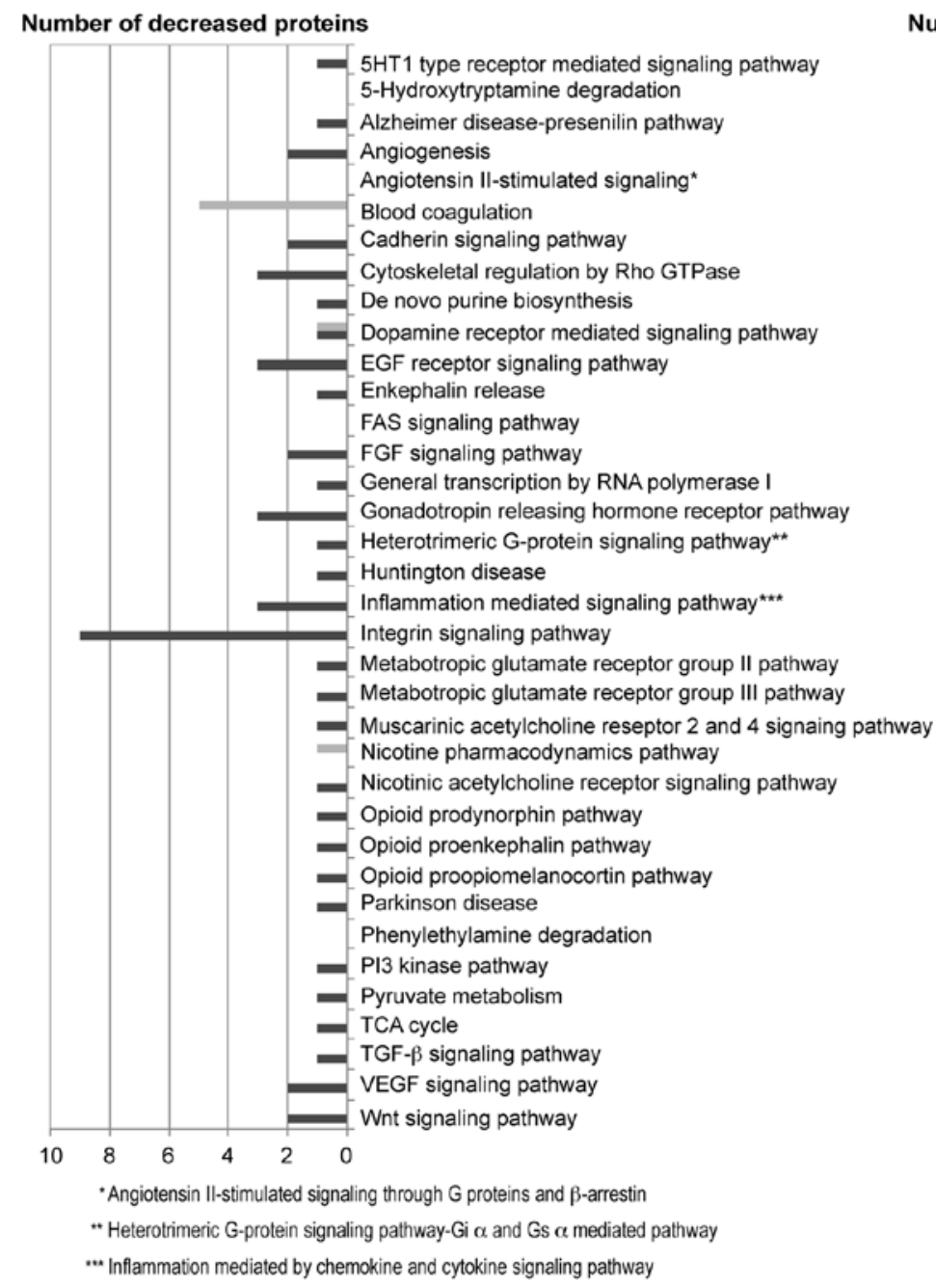

Number of increased proteins

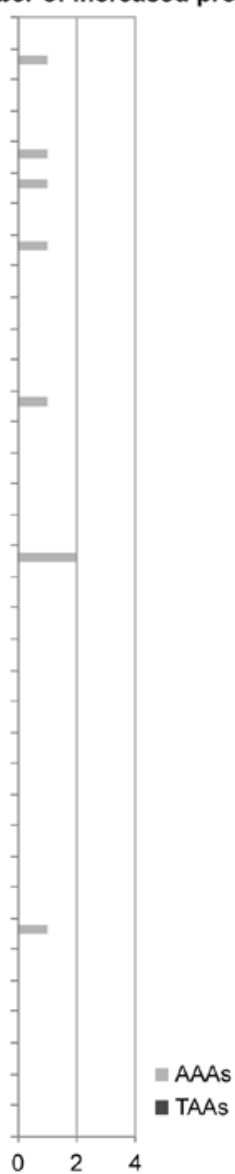

Figure 3. Pathway analysis of 55 and 68 proteins unique to abdominal aortic aneurysms (AAAs) and thoracic aortic aneurysms (TAAs) using the PANTHER pathway system. Proteins with increased expression have an isobaric tag for relative and absolute quantitation (iTRAQ) ratio of $\geq 1.0$, whereas those with decreased expression have an iTRAQ ratio of $<1.0$ (AAAs vs. NA tissues).

ANOVA using Genesis software. Consequently, 58 proteins, including plasminogen (AAAs vs. TAAs, $\mathrm{p}=5.21 \mathrm{E}-04$ ), moesin $(\mathrm{p}=5.24 \mathrm{E}-04)$, glyceraldehyde-3-phosphate dehydrogenase $(p=0.0014), \alpha-2$-macroglobulin $(p=0.0029)$, apolipoprotein B-100 ( $\mathrm{p}=0.0032)$, basement membranespecific heparan sulfate proteoglycan core protein $(\mathrm{p}=0.0041)$ and TNC $(\mathrm{p}=0.0044)$ showed significantly distinct expression patterns between the AAAs and TAAs. Furthermore, the PANTHER overrepresentation test revealed that some of the 58 proteins were significantly involved in pathways, such as plasminogen activation cascade $[p=4.11 \mathrm{E}-5$; fibrinogen $\alpha$ chain (iTRAQ ratio, 0.45, AAAs vs. NA tissues; iTRAQ ratio, 3.24, TAAs vs. NA tissues), fibrinogen $\beta$ chain (0.43:4.86), fibrinogen $\gamma$ chain $(0.44: 3.89)$, plasminogen $(0.76: 2.29)]$, blood coagulation $[\mathrm{p}=1.13 \mathrm{E}-04 ; \alpha-2$-macroglobulin (0.80:1.64), fibrinogen $\alpha$ chain (0.45:3.24), fibrinogen $\beta$ chain (0.43:4.86), fibrinogen $\gamma$ chain (0.44:3.89), plasminogen (0.76:2.29)], and integrin signaling pathway $[\mathrm{p}=3.80 \mathrm{E}-04$; type XIV collagen $\alpha 1$ chain (1.25:0.48), type XVIII collagen $\alpha 1$ chain (1.26:0.43), filamin-A (1.19:0.48), type IV collagen $\alpha 2$ chain (1.33:0.52), type XII collagen $\alpha 1$ chain (1.15:0.63), vinculin (1.03:0.50), type VI collagen $\alpha 1$ chain (1.00:0.69)]. In general, protein expression in the blood coagulation and the plasminogen activation cascade was decreased in the AAAs, whereas it was increased in the TAAs compared with the adjacent NA tissues. On the other hand, protein expression in the integrin signaling pathway was increased in the AAAs, whereas it was decreased in the TAAs compared with the adjacent NA tissues. The dendrogam based on the expression patterns of the 58 proteins demonstrated similar expression patterns of each protein within the AAA or TAA patient groups, while there were distinct expression patterns of each protein between the AAA and TAA patient groups (Fig. 5).

Verification of distinct expression patterns of TNC between AAAs and TAAs with other aneurysmal samples by western blot analysis. The matricellular protein, TNC, is upregulated under pathological conditions, such as myocardial infarction, cardiac fibrosis, atherosclerosis and aneurysms, often being associated with tissue injury and inflammation (17). It has been reported that TNC is upregulated in both AAAs $(18,19)$ and TAAs $(20,21)$. However, in the present study, as mentioned above, TNC belonged to the 58 proteins which showed distinct expression patterns in the AAAs and TAAs among the 

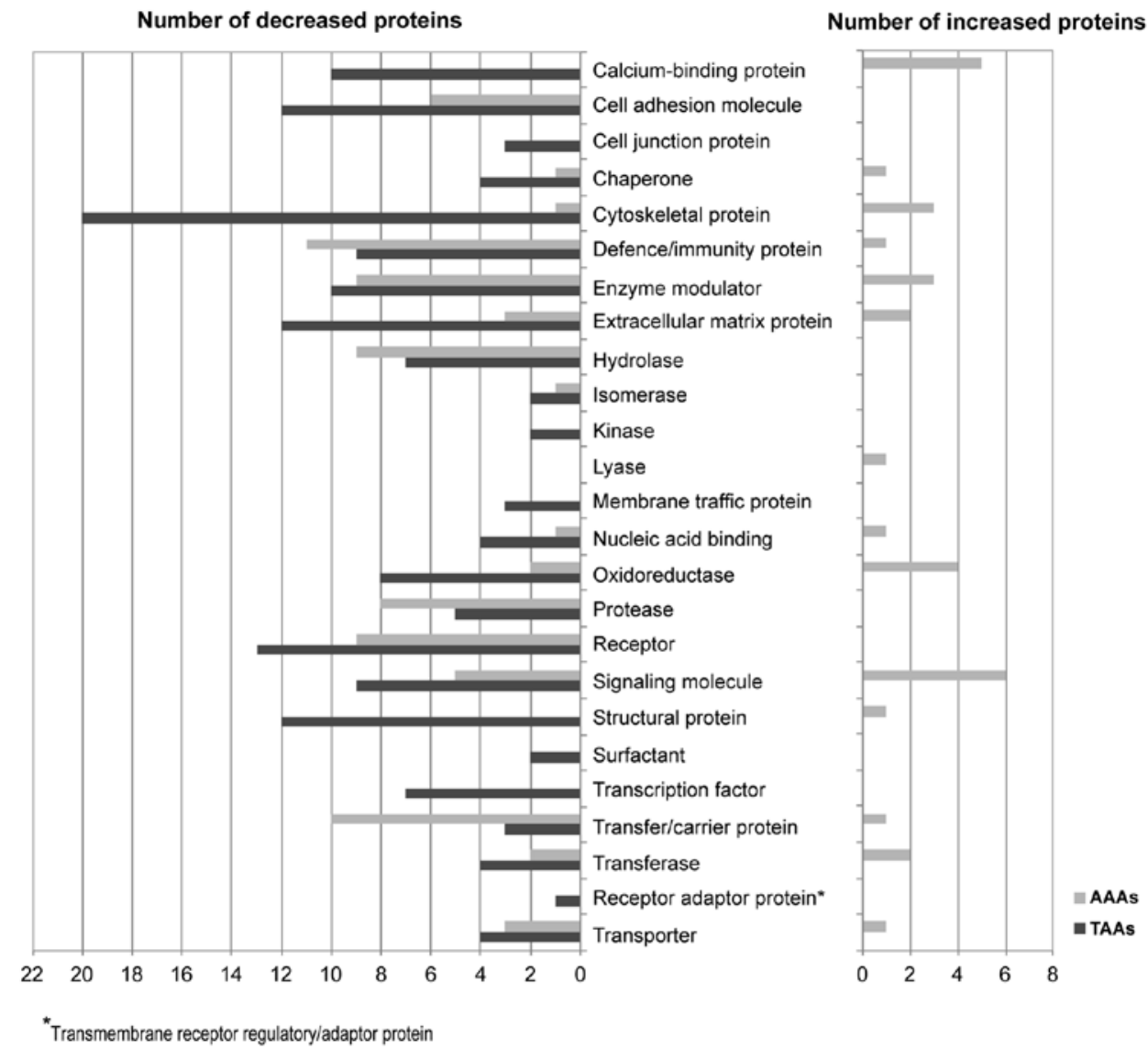

Figure 4. Classification analysis of 55 and 68 proteins unique to abdominal aortic aneurysms (AAAs) and thoracic aortic aneurysms (TAAs) using the PANTHER pathway system. Proteins with increased expression have an iTRAQ ratio of $\geq 1.0$, whereas those with decreased expression have an iTRAQ ratio of $<1.0$ (AAs vs. NA tissues). NA, normal aorta.

121 proteins; namely, its iTRAQ ratio was 0.93 in the AAAs (AAAs vs. NA tissues), whereas the iTRAQ ratio was 1.86 in the TAAs (TAAs vs. NA tissues). Thus, the expression patterns of TNC in the AAA and TAA tissues were verified with another 10 AAA and 10 TAA tissues by western blot analysis compared with those in corresponding adjacent NA tissues (Fig. 6). Consequently, we confirmed an increased level of TNC in the TAA tissues compared with the adjacent NA tissues (Fig. 6B); however, the expression level of TNC in the AAAs did not differ significantly from that in the adjacent NA tissues (Fig. 6A).

\section{Discussion}

Different features in embryonic origin, vessel structure and stiffness, immune response, the intercellular signaling pathway and proteolytic activity between the abdominal aorta and thoracic aorta contribute to the distinct symptom onsets and pathological states in AAAs and TAAs $(3,22)$. The identification of the broad range of proteomic differences between AAAs and TAAs would be valuable for their diagnosis and therapy. In this study, using an approach with iTRAQ labeling followed by nano-LC-MALDI-TOF/TOF-MS/MS analysis, proteins with differential expression in AAAs and TAAs and their adjacent NA tissues were examined. Proteomic profiles of AAAs and TAAs were then compared. Consequently, as distinct pathways activated between the AAAs and TAAs, we revealed the downregulation of the blood coagulation and plasminogen activation cascade in the AAAs and their upregulation in the TAAs, as well as the downregulation of the integrin signaling pathway in the TAAs and its upregulation in AAAs.

It is of interest that the proteins with the greatest decrease and increase in expression in the AAAs (Table III) and TAAs (Table IV), respectively, were fibrinogen $\beta, \gamma$ and $\alpha$ chains. A number of studies have been carried out on the association of plasma D-dimer (fibrinogen degradation product) with the presence of AAAs (23-25) and TAAs (26). However, to the best of our knowledge, there are only a few studies available on the finding of increased levels of fibrinogens in aortic aneurymal tissues $(26,27)$. The increased amounts of fibrinogens in TAAs are consistent with our previous results for the expression levels of fibrinogens in CTAs compared with those in adjacent NA tissues (8). However, the iTRAQ ratios of fibrinogen $\beta, \gamma$ and $\alpha$ chains in CAAs compared with those in adjacent NA tissues were $1.39,1.27$ and 1.17, respectively, indicating relatively increased levels of fibrinogens in CAA tissues (8). The reason for different levels of fibrinogens in AAA and CAA tissues compared with the levels in corresponding adjacent NA tissues remains to be determined. The different levels may be due to the distinct characteristics of AAAs and CATs. Further analyses are required.

In the present study, we observed some proteins in the integrin signaling pathway with a decreased expression in TAAs, but with an increased expression in AAAs compared with corresponding adjacent NA tissues. These results coincide 
0.0

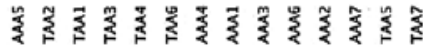
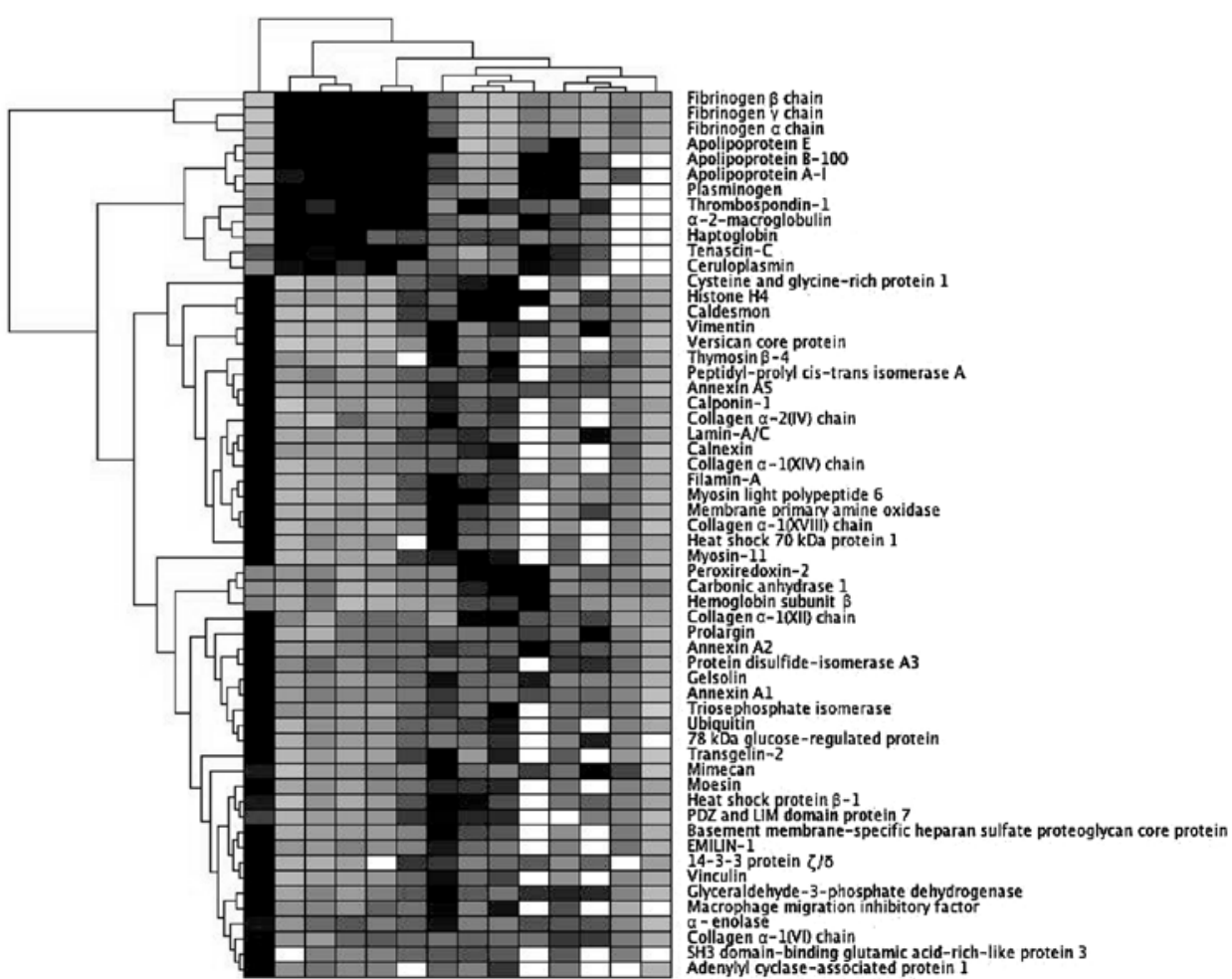

Figure 5. Hierarchical clustering analysis of the distinct expressed 58 proteins among the 121 proteins identified which were in common in abdominal aortic aneurysms (AAAs) and thoracic aortic aneurysms (TAAs) by Genesis software. The dendrogram displays two-way hierarchical clustering analyses of the distinctly expressed 58 proteins among the 121 proteins identified which were in common in at least 5 samples within 7 AAAs (\#AAA1-\#AAA7) and 7 TAAs (\#TAA1-\#TAA7) compared with corresponding adjacent normal aorta (NA) tissues. Differences in isobaric tag for relative and absolute quantitation (iTRAQ) ratios are indicated by color code. Darker black spectra indicate more increased expression, whereas lighter grey spectra indicate more decreased expression compared with corresponding adjacent NA tissues. White squares indicate no detection of indicated proteins in corresponding samples.
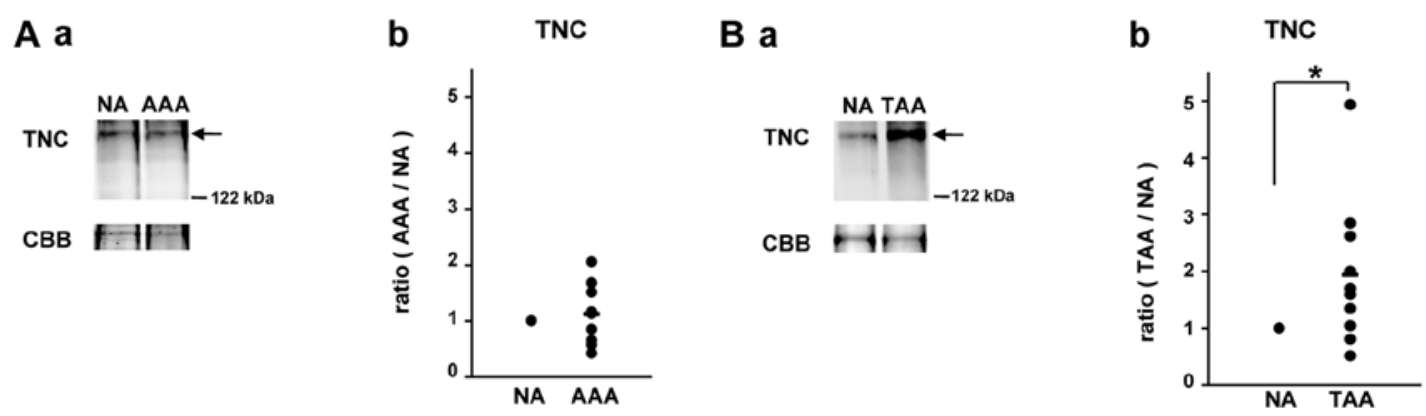

Figure 6. Verification of distinct expression patterns of tenascin-C (TNC) in abdominal aortic aneurysm (AAA) and thoracic aortic aneurysm (TAA) tissues using another $10 \mathrm{AAA}$ and $10 \mathrm{TAA}$ tissues by western blot analysis. (A-a) Western blot analysis with anti-TNC in another 10 AAAs compared with adjacent normal aorta (NA) tissues. Representative blots from patient \#AAA10 are shown. To confirm equal levels of proteins per lane, non-specific proteins stained with Coomassie brilliant blue (CBB) are shown. (b) Densitometric analyses in triplicate experiments for TNC with another 10 AAAs. The intensity of the TNC band that reacted with the antibody was measured. Ratio of expression of TNC in AAAs compared with that in adjacent NA tissues (1.0) was calculated. (B-a) Western blot analysis with anti-TNC in another 10 TAAs compared with that in adjacent NA tissues. Representative blots from patient \#TAA10 are shown. To confirm equal levels of proteins per lane, non-specific proteins stained with CBB are shown. (B-b) Densitometric analyses in triplicate experiments for TNC with another 10 TAAs. The intensity of the TNC band that reacted with the antibody was measured. Ratio of expression of TNC in TAAs compared with that in adjacent NA tissues (1.0) was calculated. " $\mathrm{p}<0.05$.

with the results of our previous study, showing the decreased expression of the integrin signaling pathway in CTAs and the increased expression of the pathway in CAAs (8). Intriguingly, Shen et al (28) demonstrated that mice with targeted deletion of ILK, one of the crucial molecules in the integrin signaling pathway, in the VSMCs generate TAAs and the marked disruption of the structural organization in the arterial tunica media by aberrant integrin signaling, including abnormal local- 
izacion of myocardin-related transcription factors (MRTFs), a reduced amount of F-actin and impaired RhoA activation in ILK-deficient VSMCs. On the other hand, the increased expression of integrin $\alpha_{\mathrm{v}}$ was actually observed at the site of AAA rupture (29). Zheng et al (30) showed that osteopontin induces autophagy through the activation of the integrin/CD44 and p38 mitogen-activated protein kinase (MAPK) signaling pathways in VSMCs, which is associated with the incidence of AAAs.

Didangelos et al (7) reported results of proteomic analysis of the ECM of human AAAs compared with that of control samples from patients without connective tissue disorders. They identified $80 \mathrm{ECM}$ proteins with extraction using $0.5 \mathrm{M} \mathrm{NaCl}$ extraction buffer and $117 \mathrm{ECM}$ proteins with the extraction using $4 \mathrm{M}$ guanidine buffer, with the difference in relative protein abundance between AAAs and controls being determined using spectral counts. Thirty-four proteins among the 80 proteins and 21 proteins among the 117 proteins were found to be common in the 176 differentially expressed proteins in AAAs identified in the present study. Biglycan and type I collagen $\alpha 1$ and $\alpha 2$ chains in AAA tissues were upregulated by $>1$.3-fold, whereas versican was downregulated by $<0.77$-fold in both their experiments and the present study (AAA samples vs. control samples).

Recently, we reported that the kallistatin level in presurgical sera of both AAA and TAA patients and the $\alpha$-2-macroglobulin level in pre-surgical sera of TAA patients were increased compared with those in the post-surgical sera of the corresponding patients (31). In the present study, kallistatin was not identified as a differentially expressed protein in both aneurysmal tissues, whereas $\alpha$-2-macroglobulin expression was increased in the TAAs (1.64-fold) compared with that in adjacent normal tissues. Therefore, these results demonstrated that $\alpha$-2-macroglobulin was increased not only in the serum of patients with TAAs but also in TAA tissues. $\alpha$-2-macroglobulin plays a role in the inhibition and clearance of active proteases, including all of the 4 major classes of endopeptidases in tissue fluids (32). Since MMP activity is high in AAs and positively correlates with aneurysmal size (33), the upregulation of endogenous MMP inhibitors, such as $\alpha$-2-macroglobulin in TAAs may be for the prevention of further TAA progression.

In the present study, we demonstrate the increased level of TNC in TAAs, but not in AAAs compared with adjacent normal tissues. The finding of the increased expression of TNC in TAAs is consistent with the results of previous studies $(20,21)$. However, our results regarding the expression of TNC in AAAs are not consistent with those of previous studies, showing the upregulation of TNC in AAA tissues $(18,19,34)$. The reason for this inconsistency is not known. However, Kimura et al (19) reported that the expression level of TNC did not correlate with the AAA diameter in human AAAs. This suggests that AAA tissues have heterogeneity in pathology.

In conclusion, our data reveal opposite expression patterns of proteins in the blood coagulation and plasminogen activation cascade and integrin signaling pathway between AAAs and TAAs. These distinct alterations of their proteomes may lead to the difference in the pathology between AAAs and TAAs.

\section{Acknowledgements}

We thank Yasuko Sonoyama for sample collections. This study was supported in part by Grants-in-Aid for Scientific Research
(22590063 to K.M.) from the Ministry of Education, Culture, Sports, Science and Technology of Japan.

\section{References}

1. Guo DC, Papke CL, He R and Milewicz DM: Pathogenesis of thoracic and abdominal aortic aneurysms. Ann NY Acad Sci 1085: 339-352, 2006.

2. Kuivaniemi H, Platsoucas CD and Tilson MD III: Aortic aneurysms: an immune disease with a strong genetic component. Circulation 117: 242-252, 2008.

3. Ruddy JM, Jones JA, Spinale FG and Ikonomidis JS: Regional heterogeneity within the aorta: relevance to aneurysm disease. J Thorac Cardiovasc Surg 136: 1123-1130, 2008.

4. Henderson EL, Geng YJ, Sukhova GK, Whittemore AD, Knox J and Libby P: Death of smooth muscle cells and expression of mediators of apoptosis by $\mathrm{T}$ lymphocytes in human abdominal aortic aneurysms. Circulation 99: 96-104, 1999.

5. Tang PC, Yakimov AO, Teesdale MA, et al: Transmural inflammation by interferon-gamma-producing $\mathrm{T}$ cells correlates with outward vascular remodeling and intimal expansion of ascending thoracic aortic aneurysms. FASEB J 19: 1528-1530, 2005.

6. Absi TS, Sundt TM III, Tung WS, et al: Altered patterns of gene expression distinguishing ascending aortic aneurysms from abdominal aortic aneurysms: complementary DNA expression profiling in the molecular characterization of aortic disease. $\mathrm{J}$ Thorac Cardiovasc Surg 126: 344-357, 2003.

7. Didangelos A, Yin X, Mandal K, et al: Extracellular matrix composition and remodeling in human abdominal aortic aneurysms: a proteomics approach. Mol Cell Proteomics 10: M111.008128, 2011.

8. Matsumoto K, Maniwa T, Tanaka T, Satoh K, Okunishi H and Oda T: Proteomic analysis of calcified abdominal and thoracic aortic aneurysms. Int J Mol Med 30: 417-429, 2012.

9. Matsumoto K, Satoh K, Maniwa T, Araki A, Maruyama R and Oda T: Noticeable decreased expression of tenascin-X in calcific aortic valves. Connect Tissue Res 53: 460-468, 2012.

10. Ross PL, Huang YN, Marchese JN, et al: Multiplexed protein quantitation in Saccharomyces cerevisiae using amine-reactive isobaric tagging reagents. Mol Cell Proteomics 3: 1154-1169, 2004.

11. Matsumoto K: Phosphorylation of extracellular matrix tenascin-X detected by differential mass tagging followed by nanoLC-MALDITOF/TOF-MS/MS using ProteinPilot software. Connect Tissue Res 53: 106-116, 2012.

12. Shilov IV, Seymour SL, Patel AA, et al: The Paragon Algorithm, a next generation search engine that uses sequence temperature values and feature probabilities to identify peptides from tandem mass spectra. Mol Cell Proteomics 6: 1638-1655, 2007.

13. Mi H, Muruganujan A and Thomas PD: PANTHER in 2013: modeling the evolution of gene function, and other gene attributes, in the context of phylogenetic trees. Nucleic Acids Res 41: D377-D386, 2013.

14. Sturn A, Quackenbush J and Trajanoski Z: Genesis: cluster analysis of microarray data. Bioinformatics 18: 207-208, 2002.

15. Hu HD, Ye F, Zhang DZ, Hu P, Ren H and Li SL: iTRAQ quantitative analysis of multidrug resistance mechanisms in human gastric cancer cells. J Biomed Biotechnol 2010: 571343, 2010.

16. Magharious M, D'Onofrio PM, Hollander A, Zhu P, Chen J and Koeberle PD: Quantitative iTRAQ analysis of retinal ganglion cell degeneration after optic nerve crush. J Proteome Res 10: 3344-3362, 2011.

17. Imanaka-Yoshida $\mathrm{K}$ : Tenascin- $\mathrm{C}$ in cardiovascular tissue remodeling: from development to inflammation and repair. Circ J 76: 2513-2520, 2012.

18. Satta J, Soini Y, Pöllänen R, Pääkkö P and Juvonen T: Tenascin expression is associated with a chronic inflammatory process in abdominal aortic aneurysms. J Vasc Surg 26: 670-675, 1997.

19. Kimura T, Yoshimura K, Aoki H, et al: Tenascin-C is expressed in abdominal aortic aneurysm tissue with an active degradation process. Pathol Int 61: 559-564, 2011.

20. Majumdar R, Miller DV, Ballman KV, et al: Elevated expressions of osteopontin and tenascin $\mathrm{C}$ in ascending aortic aneurysms are associated with trileaflet aortic valves as compared with bicuspid aortic valves. Cardiovasc Pathol 16: 144-150, 2007.

21. Trescher K, Thometich B, Demyanets S, et al: Type A dissection and chronic dilatation: tenascin-C as a key factor in destabilization of the aortic wall. Interact Cardiovasc Thorac Surg 17: 365-370, 2013. 
22. Isselbacher EM: Thoracic and abdominal aortic aneurysms Circulation 111: 816-828, 2005.

23. Parry DJ, Al-Barjas HS, Chappell L, Rashid T, Ariëns RA and Scott DJ: Haemostatic and fibrinolytic factors in men with a small abdominal aortic aneurysm. Br J Surg 96: 870-877, 2009.

24. Takagi H, Manabe H, Kawai N, Goto S and Umemoto T: Plasma fibrinogen and D-dimer concentrations are associated with the presence of abdominal aortic aneurysm: a systematic review and meta-analysis. Eur J Vasc Endovasc Surg 38: 273-277, 2009.

25. Golledge J, Muller R, Clancy P, McCann M and Norman PE: Evaluation of the diagnostic and prognostic value of plasma D-dimer for abdominal aortic aneurysm. Eur Heart J 32: 354-364, 2011.

26. Yuan SM, Shi YH, Wang JJ, Lü FQ and Gao S: Elevated plasma D-dimer and hypersensitive C-reactive protein levels may indicate aortic disorders. Rev Bras Cir Cardiovasc 26: 573-581, 2011.

27. Ando T, Nagai K, Chikada M, et al: Proteomic analyses of aortic wall in patients with abdominal aortic aneurysm. J Cardiovasc Surg (Torino) 52: 545-555, 2011.

28. Shen D, Li J, Lepore JJ, Anderson TJ, et al: Aortic aneurysm generation in mice with targeted deletion of integrin-linked kinase in vascular smooth muscle cells. Circ Res 109: 616-628, 2011.
29. Choke E, Cockerill GW, Dawson J, et al: Increased angiogenesis at the site of abdominal aortic aneurysm rupture. Ann NY Acad Sci 1085: 315-319, 2006

30. Zheng YH, Tian C, Meng Y, et al: Osteopontin stimulates autophagy via integrin/CD44 and p38 MAPK signaling pathways in vascular smooth muscle cells. J Cell Physiol 227: 127-135, 2012.

31. Satoh K, Maniwa T, Oda T and Matsumoto K: Proteomic profiling for the identification of serum diagnostic biomarkers for abdominal and thoracic aortic aneurysms. Proteome Sci 11: 27, 2013.

32. Armstrong PB and Quigley JP: Alpha2-macroglobulin: an evolutionarily conserved arm of the innate immune system. Dev Comp Immunol 23: 375-390, 1999.

33. Zarins CK, Runyon-Hass A, Zatina MA, Lu CT and Glagov S: Increased collagenase activity in early aneurysmal dilatation. J Vasc Surg 3: 238-248, 1986.

34. Paik DC, Fu C, Bhattacharya J and Tilson MD: Ongoing angiogenesis in blood vessels of the abdominal aortic aneurysm. Exp Mol Med 36: 524-533, 2004. 